\title{
Correção das deficiências transversas e ântero- posteriores da maxila em pacientes adultos
}

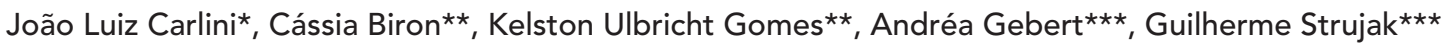

\section{Resumo}

Objetivo: a finalidade deste trabalho é avaliar os resultados de tratamento de pacientes com desarmonias esqueléticas Classe III e discrepâncias transversas, tratados com a técnica de expansão maxilar assistida cirurgicamente, associada à mecânica de tração reversa. Metodologia: Foram avaliados 10 pacientes portadores de Classe III esquelética e discrepância transversal, submetidos à expansão maxilar assistida cirurgicamente e ao uso de máscara facial. A avaliação das alterações ortodônticas e ortopédicas foram realizadas por meio dos exames clínico e radiográfico. Conclusão: os resultados clínicos e radiográficos demonstraram eficiência nestes procedimentos, evitando nova cirurgia de maxila, diminuindo a morbidade do tratamento e o seu custo.

Palavras-chave: Tração maxilar. Discrepância transversa da maxila. Cirurgia ortognática.

\section{INTRODUÇÃO}

A tração reversa de maxila constitui uma mecânica ortopédica, obtida através de um aparelho extrabucal removível, denominado máscara facial. Esta é utilizada para reposicionar a maxila em direção anterior, portanto, estando indicada para correção da má oclusão Classe III com retrognatismo maxilar. A associação da expansão cirúrgica de maxila e tração reversa permite movimentar a maxila no sentido ântero-posterior e transverso, simultaneamente, descartando a necessidade de duas intervenções cirúrgicas (Expansão cirúrgica da maxila e posterior avanço maxilar) para a correção da Classe III.

A disjunção palatina é rápida e produz uma abertura na sutura palatina mediana. O parafuso deve produzir $1 \mathrm{~mm}$ de expansão a cada volta completa. As bandas ortodônticas são cimentadas nos molares e pré-molares. O aparelho é ativado diretamente na boca do paciente, duas vezes ao dia. No período inicial do movimento, ocorre uma inclinação vestibular dos dentes, seguida por um período secundário de abertura da sutura, evidenciado por um diastema interincisivos, o qual aumenta progressivamente, à medida que há expansão. $O$ período de contenção, após a expansão desejada, deve ser de, no mínimo, 6 meses, para que não haja risco de recidiva. Ao longo deste período de consolidação, ocorre uma aposição óssea nas margens da sutura mediana ${ }^{6}$.

Os pacientes com deficiência do terço médio de face e prognatismo mandibular requerem estratégias combinadas, tração reversa e expansão maxilar ${ }^{10}$.

Aproximadamente 5\% da população branca exibem má oclusão esquelética de Classe III, caracterizada por protrusão mandibular, retrusão

\footnotetext{
* Cirurgião Bucomaxilofacial do CAIF (Centro de Atendimento Integral ao Fissurado Lábio-palatal). Professor de Cirurgia e Traumatologia Bucomaxilofacial da UFPR.

** Cirurgiões Bucomaxilofacial do CAIF.

*** Estagiários em Cirurgia Bucomaxilofacial do CAIF.
} 
maxilar ou a combinação destes dois fatores. Segundo os autores, o padrão facial sofre interferência em relação à direção da força a ser aplicada para a protração maxilar. A força horizontal pode causar rotação anti-horária da maxila, resultando em mordida aberta. Em contrapartida, a força de protração direcionada para baixo pode produzir um reposicionamento do complexo nasomaxilar e das estruturas adjacentes, alongando a face ${ }^{11}$.

Os pacientes com má oclusão de Classe III possuem deficiência maxilar, prognatismo mandibular ou a combinação dos dois fatores e, freqüentemente, apresentam deficiência transversa da maxila ${ }^{12}$.

Foi identificado que 32 a $43 \%$ dos pacientes com má oclusão de Classe III possuem um crescimento recessivo da maxila ou a combinação de maxila recessiva e excesso de crescimento mandibular ${ }^{13}$.

A má oclusão de Classe III é definida pela presença de uma mandíbula larga, maxila pequena, maxila retroposicionada ou a combinação destes três fatores. Os estudos demonstraram que $42 \%$ a 60\% das más oclusões de Classe III apresentam maxila deficiente ou retruída, em combinação com uma mandíbula normal ou levemente prognata ${ }^{7}$.

A relação de Classe III foi classificada em dentária, esquelética ou de posição. As esqueléticas e dentárias estão relacionadas a problemas de crescimento, tamanho, forma e proporção dentro do complexo maxilofacial. As anomalias de posição se traduzem por um distúrbio funcional. Nesta, o fechamento mandibular se inicia normalmente, mas a partir de um contato prematuro, há um desvio, levando a uma oclusão de conveniência Classe III. Nestes casos não existem problemas esqueléticos, discrepância entre tamanho de maxila e mandíbula, podendo ser chamada de pseudoclasse III ou pseudoprognatismo. A etiologia está ligada a fatores gerais (distúrbios hormonais, fissura lábio palatina, traumatismos), fatores locais (problemas de postura mandibular, perda prematura dos primeiros molares, distúrbios na erupção dos incisivos, hipertrofia de adenóides e tonsilas palatinas) e fatores hereditários ${ }^{1}$.

Os problemas de subdesenvolvimento maxilar requerem como tratamento o movimento anterior da maxila, movimentação dentária anterior e melhora do perfil. Com este objetivo, o autor sugere, até a adolescência, o uso da máscara facial de Delaire, a qual pode ser associada em conjunto, ou mais tardiamente, a um aparelho de expansão rápida da maxila, pois freqüentemente é encontrado um estreitamento do arco superior, com mordida cruzada posterior bilateral ${ }^{2}$.

A expansão rápida de maxila é indicada com abertura da sutura palatina mediana, nos casos de má oclusão de Classe III e deficiências maxilares reais ou relativas ${ }^{4}$.

No tratamento de pacientes jovens, portadores de má oclusão Classe III, recomenda-se o uso do expansor rápido de maxila e máscara facial ortopédica, os quais, em combinação, produzem uma resposta mais rápida. $\mathrm{O}$ efeito da expansão, no contexto da terapia da máscara facial, é abrir o sistema de sutura maxilar, possibilitando a ocorrência do efeito ortopédico, fazendo com que os ajustes suturais ocorram imediatamente ${ }^{8}$.

O tratamento recomendado para pacientes jovens portadores de Classe III é a expansão palatina e a protração maxilar. Segundo o autor ${ }^{14}$, os efeitos da expansão palatina não ocorrem somente na sutura intermaxilar, mas em todo o complexo sutural que circunda a maxila. A expansão palatina desarticula a maxila e inicia uma resposta celular nas suturas, favorecendo a força de protração. Outra vantagem da expansão, é a correção da mordida cruzada posterior, que acompanha a má oclusão Classe III, pois em geral há crescimento transversal deficiente e relação ântero-posterior anormal da maxila e da mandíbula.

Considerando-se que as discrepâncias transversas de até $4 \mathrm{~mm}$ poderão ser compensadas com movimentos dentários, inclinando-se $1 \mathrm{~mm}$ para vestibular os molares superiores e $1 \mathrm{~mm}$ para lingual os molares inferiores, o autor ${ }^{9}$ ressalta a importância da manipulação dos modelos superior e 
inferior para diagnosticar se a discrepância transversal é real ou virtual.

A posição do arco inferior, à frente do superior, na discrepância basal de Classe III, exige um diagnóstico diferencial entre deficiência maxilar transversa real ou relativa. $O$ diagnóstico pode ser efetuado mediante a análise dos modelos de gesso em intercuspidação Classe I. A mordida cruzada posterior na posição corrigida caracteriza uma deficiência real ${ }^{5}$.

A expansão orto-cirúrgica é recomendada em pacientes com idade acima de 16 anos com discrepância transversa de maxila de $5 \mathrm{~mm}$ ou mais. A necessidade deste procedimento é determinada pela observação dos modelos em sua posição ântero-posterior e/ou medindo-se as relações transversas (cúspide mesiopalatina do primeiro molar superior em relação à fossa central dos primeiro molar inferior ou cúspide palatina do $1^{\circ}$ pré-molar superior em relação à face marginal distal do primeiro pré-molar inferior). A mordida cruzada posterior deve ser analisada no modelo de estudo, manipulando os modelos em relação de Classe I de caninos e corrigindo a linha média. Se permanecer cruzada, a mordida posterior trata-se de uma verdadeira discrepância esquelética. Caso contrário, considera-se uma mordida cruzada virtual. Se houver inclinações dentárias, o ortodontista deve, para solucionar o problema transverso, corrigir estas inclinações. Se houver discrepância esquelética, em pacientes adultos, é necessária uma expansão orto-cirúrgica para corrigir o problema. Juntamente com a discrepância transversal, pode haver uma má oclusão Classe III, causada pela deficiência ântero-posterior de maxila ( 1 a $3 \mathrm{~mm}$ ). Em geral, isto é corrigido com uso de elásticos, imediatamente após a osteotomia maxilar para expansão. $\mathrm{O}$ aparelho disjuntor deve possuir somente apoio dentário, para permitir um ótimo acesso cirúrgico e evitar uma possível necrose de palato. Esta última consideração é muito importante porque o maior suprimento sangüíneo do segmento ósseo-dentário da maxila que será expandida provém da mucosa palatina. A dieta deverá ser líquida-pastosa por 5 a 6 semanas após a cirurgia, até uma razoável união óssea estar estabelecida. O paciente deverá ser informado que o procedimento resultará em um grande diastema na linha média, que estará presente por 2 a 4 meses, sendo posteriormente fechado ortodonticamente. Após 5 dias, o aparelho deve ser ativado $1 / 4$ de volta, duas vezes ao dia, em intervalos apropriados até a expansão completa. É importante não começar o fechamento do diastema interincisivos até que se tenha a evidência radiográfica da formação óssea entre os incisivos, fato que geralmente ocorre entre 8 e 12 semanas após a cirurgia. Quando o movimento ântero-posterior da maxila for desejado, elásticos pesados podem ser usados após a cirurgia (até 8 semanas), desempenhando um movimento de 2 a $3 \mathrm{~mm}$ de avanço da maxila. A estabilização é mantida por 2 meses ${ }^{3}$.

Dessa forma, o objetivo desse trabalho é avaliar os resultados de pacientes com desarmonias esqueléticas Classe III e discrepâncias transversais, tratados com a técnica de expansão maxilar assistida cirurgicamente, associada à mecânica de tração reversa.

\section{MATERIAL E MÉTODOS}

O estudo foi realizado com 10 pacientes adultos, com idade variando de 25 a 34 anos, sendo 7 pacientes do gênero masculino e 3 do gênero feminino. Todos eram portadores de Classe III esquelética e discrepância transversal real. Seis pacientes apresentavam retrognatismo maxilar e 3 pacientes apresentavam mau posicionamento mandibular.

Para potencializar o efeito ortopédico da tração reversa, recomendou-se a instalação do aparelho expansor tipo Hyrax, que recebeu ganchos soldados na vestibular de caninos e pré-molares. Nestes ganchos, foram encaixados os elásticos que fazem a ligação entre o expansor e a máscara facial. Os elásticos utilizados promoveram uma força de $500 \mathrm{~g}$ de cada lado e o paciente foi orientado a usar a máscara facial por 16 a 18 horas ao dia, 
até a sobrecorreção da deficiência. A média de uso da máscara foi de 4 meses e durante seu uso foi realizada a expansão da maxila.

Com o paciente sob anestesia geral, foi infiltrado anestésico local associado a 1:200.000 de epinefrina no fundo de vestíbulo maxilar. A incisão foi realizada no fundo de vestíbulo com uma lâmina $n^{0} 15$, extendendo-se do primeiro molar ao primeiro molar contralateral. Os tecidos moles foram destacados subperiostalmente, expondo a porção superior e lateral da maxila, a fossa nasal e a abertura piriforme. $\mathrm{O}$ descolamento foi tunelizado para posterior, em direção à junção pterigomaxilar. A osteotomia da parede lateral da maxila foi determinada $5 \mathrm{~mm}$ acima dos ápices dentários. A osteotomia da parede lateral foi realizada com serra reciprocante, iniciando na abertura piriforme e inclinando-se inferiormente em direção aos molares, para minimizar a necessidade da separação pterigomaxilar com cinzéis. A parede mais posterior e lateral da maxila foi osteotomizada com um cinzel curvo, separando a maxila do processo pterigóide. Esta técnica foi utilizada em ambos os lados. A linha média foi separada, inicialmente, com serra reciprocante e com um cinzel reto, respeitando as raízes dos incisivos. Enquanto esta osteotomia era realizada, um dedo indicador era colocado no palato, para sentir a passagem do cinzel, evitando lesionar a mucosa palatina. A osteotomia Le Fort I era realizada por completo, evitando somente o rebaixamento e soltura da maxila (downfracture). Nesta fase, o aparelho expansor foi ativado para confirmar se a maxila estava expandindo simetricamente. Em seguida, retornou-se o parafuso totalmente e realizou-se a sutura com fio de poligalactina 4.0. Realizou-se uma bandagem externa com micropore e foi recomendado o uso de compressas com gelo no local, antibioticoterapia com cefalotina (24horas), analgesia com paracetamol e higiene bucal. Após 7 dias de cirurgia, iniciou-se a ativação do aparelho tipo Hyrax, ativando $1 / 2$ volta ao dia do parafuso, divido em $1 / 4$ de volta pela manhã e $1 / 4$ de volta à tarde. Concomitante à expan- são, foi instalada a máscara facial, que deveria ser utilizada por 16 a 18 horas ao dia, com elásticos de $500 \mathrm{mg}$ de cada lado (Fig. 2, 3). O período médio de uso do Hyrax e da máscara facial foi de 4 meses. Após este período, o paciente foi liberado para o tratamento ortodôntico. Recomenda-se que, antes do procedimento na maxila, seja instalado aparelho ortodôntico fixo no arco inferior, para descompensar os dentes inferiores e estabilizá-los com arcos retangulares. Nesta situação se consegue a condição real dos movimentos da maxila no sentido ântero-posterior e transversal.

O acompanhamento foi realizado pelo ortodontista e pelo cirurgião, sendo que o exame clínico foi decisivo para interromper a expansão e a tração da maxila. Para mensurar o movimento ântero-posterior da maxila, toma-se como referências as bordas incisais dos incisivos centrais superiores e inferiores. Quanto ao movimento transverso, houve somente avaliação clínica.

Foram solicitadas radiografias pós-operatórias (telerradiografia de perfil e oclusal superior): a primeira, para mensurar o movimento ântero-posterior e a segunda para observar a consolidação óssea da sutura palatina.

\section{RESULTADOS}

Os resultados foram baseados na avaliação clínica e na mensuração das bordas incisais dos incisivos superiores e inferiores (Tab. 1).

Tabela 1 - Avanço da maxila.

\begin{tabular}{cc}
\hline pacientes & tração da maxila \\
\hline 1 & $6 \mathrm{~mm}$ \\
2 & $4 \mathrm{~mm}$ \\
3 & $6 \mathrm{~mm}$ \\
4 & $7 \mathrm{~mm}$ \\
5 & $5 \mathrm{~mm}$ \\
6 & $5 \mathrm{~mm}$ \\
7 & $6 \mathrm{~mm}$ \\
8 & $5 \mathrm{~mm}$ \\
9 & $7 \mathrm{~mm}$ \\
10 & $6 \mathrm{~mm}$ \\
\hline
\end{tabular}



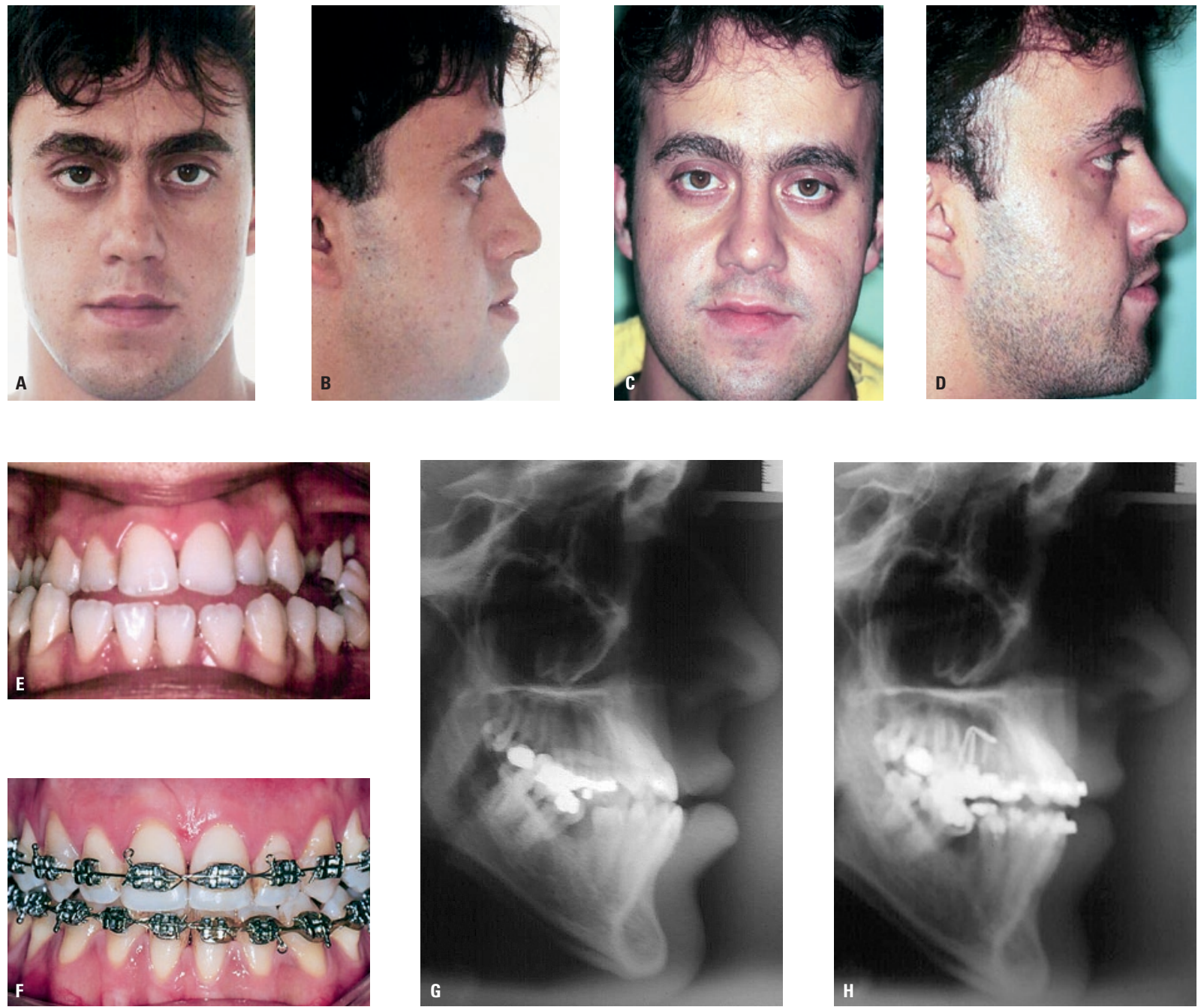

FIGURA 1 - A, B, E, G) pré-operatório e C, D, F, H) pós-operatório.

Com intuito de exemplificação, pode ser observada, nos casos clínicos apresentados (Fig. 1, 2), a aplicação da técnica proposta.

\section{DISCUSSÃO}

O tratamento não-cirúrgico dos problemas transversos e ântero-posteriores da maxila em pacientes jovens apresenta resultados satisfatórios e previsíveis ${ }^{1,2,4,6,8,10,11,12,14}$. Entretanto, quando tratase de pacientes adultos, existem algumas limitações e somente o tratamento ortodôntico não é suficiente.

O ponto mais importante desta discussão está no correto diagnóstico da discrepância transversa, visto que é fundamental a manipulação dos modelos de gesso superior e inferior em relação de Classe I dos caninos, pois somente nesta condição pode-se afirmar se a discrepância transversa da maxila é real ou virtual. Na maioria das situações, este exame é feito clinicamente na boca do paciente, induzindo o profissional ao erro. Um planejamento incorreto pode levar a uma expansão da maxila desnecessária ou a uma inclinação compensatória de dentes superiores para vestibular, impedindo uma correta oclusão dentária quando a maxila for anteriorizada ${ }^{3,5,9}$. 

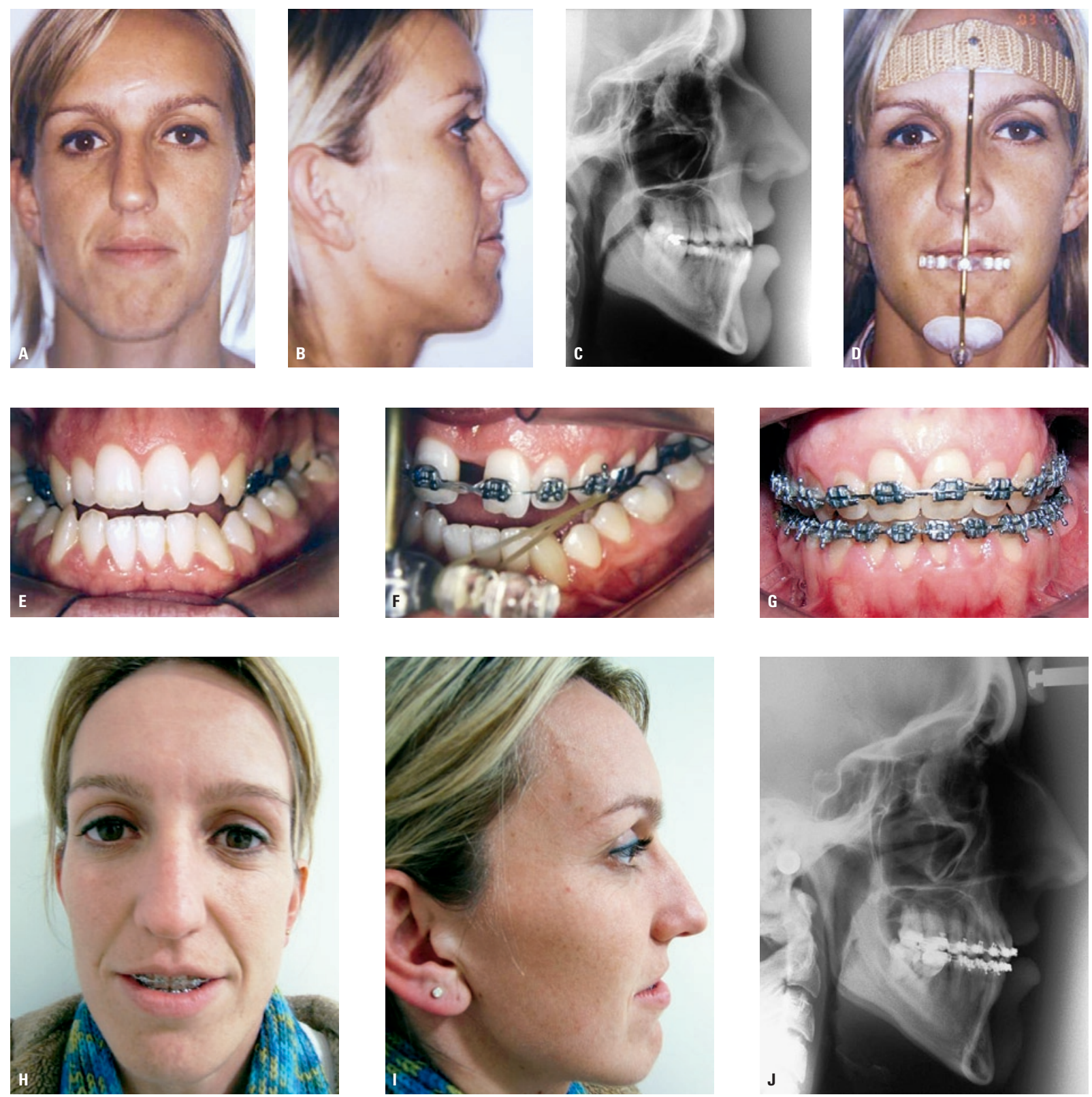

FIGURA 2 - A, B, C, E) Pré-operatório, D, F) período de ativação e G, H, I, J) pós-operatório.

Em relação aos casos tratados, o avanço da maxila foi, em média, de $5 \mathrm{~mm}$, com um máximo de $7 \mathrm{~mm}$. Em relação ao uso da máscara, os pacientes relataram dificuldade de adaptação na primeira semana e alguns queixaram-se de dor na região da articulação temporomandibular, nas primeiras horas do uso da máscara. Não foram observadas complicações trans e pós-operatórias, como san- gramento, infecções ou pseudoartrose. Especial atenção foi dada para o direcionamento do elástico, para que não ocorresse desvio assimétrico, movimento para superior ou inferior da maxila. $\mathrm{O}$ arco inferior foi importante para o correto direcionamento da maxila, através de elásticos intermaxilares orientados em Classe III.

A aplicação desta forma de tratamento está 
indicada, principalmente para os pacientes que apresentam deficiências ântero-posteriores e transversais da maxila.

Para pacientes que apresentam protrusão mandibular, esta forma de tratamento também pode ser utilizada, pois minimiza a necessidade da segunda cirurgia, além de reduzir o custo, pois não necessita do material de fixação rígida na maxila.

Teoricamente, é possível avançar a maxila mais do que foi obtido neste trabalho, pois a mesma está deslocada da base do crânio, sustentada pelos tecidos moles. Entretanto, dependerá da colaboração do paciente no uso continuo da máscara facial.

A estabilidade após a tração é feita com elásticos no arco inferior, posicionados em Classe III, e dieta líquida-pastosa, enquanto a máscara facial estiver em uso.

A maior dificuldade de aplicação desta técnica está na resistência e na colaboração do paciente em usar a máscara facial. As vantagens, bem como seus benefícios, deverão ser detalhadamente explicadas aos pacientes, destacando a redução da morbidade, redução do tempo de tratamento e a redução dos custos.

\section{CONCLUSÕES}

A utilização da técnica descrita proporciona um menor tempo de tratamento, menor custo e, principalmente, evita a necessidade de duas intervenções cirúrgicas (disjunção e avanço maxilar), solucionando, simultaneamente, o problema transverso e ântero-posterior de maxila. As desvantagens observadas são a dificuldade de adaptação no uso da máscara facial e o compromisso do paciente na sua utilização.

\section{AGRADECIMENTOS}

Agradecemos à ortodontista Dra. Karin Freund, por nos enviar estes pacientes e pela competência na condução do tratamento dos mesmos.

Maxillary anteroposterior and transverse problems correction in adult patients

\begin{abstract}
Aim: The purpose of this article is to evaluate the correction of anteroposterior and transverse maxillary deficiency in adult patients with the use of facial mask and surgically assisted RME (SARME). Methods: Reverse traction of the maxilla was applied to 10 subjects through a facial mask after a SARME. All individuals presented a Class III skeletal malocclusion. Cephalometric tracings were used to access the amount of anterior maxillary displacement during treatment. Results and Conclusion: The association of these procedures promoted transverse correction and anterior displacement of the maxilla. This approach avoided a two step correction - one for SARME and other for maxillary advancement, reducing morbidity and costs of treatment.
\end{abstract}

Key words: Maxillary traction. Transverse deficiency. Orthognathic surgery. 


\section{REFERÊNCIAS}

1. COUGHI, O. A.; MENDONÇA, M. R. de; SANTOS, E. C. A. Tratamento das maloclusões de Classe III. J. Brás. Ortodon. Ortop. Maxilar, Curitiba, v. 2, n. 11, p. 31-41, set./out. 1997.

2. COZZANI, G. Extraoral traction and Class III treatment. Am. J. Orthod. Dentofacial Orthop., St. Louis, v. 80, no. 6, p. 638650, Dec. 1981.

3. EPKER, B. N.; FISH, L. C. Dentofacial deformities: integrated orthodontic and surgical correction. Missouri: C. V. Mosby, 1986.

4. HAAS, A. J. Palatal expansion: just the beginning of dentofacial orthopedics. Am. J. Orthod. Dentofacial Orthop., St. Louis, v. 57, no. 3, p. 219-255, Mar. 1970.

5. INTERLANDI, S. Ortodontia: bases para iniciação. 3. ed. São Paulo: Artes Médicas, 1994.

6. LANGLADE, M. Terapêutica ortodôntica. 3. ed. São Paulo: Ed. Santos, 1995.

7. LEE, K. et al. A study of holografic interferometry on the initial reaction of maxillofacial complex during protraction. Am. J. Orthod. Dentofacial Orthop., St. Louis, v. 111, no. 6, p. 623632, June 1997.
8. McNAMARA JUNIOR, J. A. An orthopedic approach to the treatment of Class III malocclusion in young patients. J. Clin. Orthod., Boulder, v. 21, no. 9, p. 598-608, Sept. 1987.

9. MEDEIROS, P. J. Cirurgia ortognática para o ortodontista São Paulo: Ed. Santos, 2001.

10. MOYERS, R. E. Ortodontia. 4. ed. Rio de Janeiro: Guanabara Koogan, 1991.

11. NGAN, P. et al. Effect of protraction headgear on Class III malocclusion. Quintessence Int., Berlin, v. 23, no. 3, p. 197-207, Mar. 1992.

12. STAGGERS, J. A.; GERMANE, N.; LEGAN, H. L. Clinical considerations in the use of protraction headgear. J. Clin. Orthod. Boulder, v. 26, no. 2, p. 87-91, Feb. 1992.

13. TAKADA, K.; PETDACHAI, S.; SAKUDA, M. Changes in dentofacial morphology in skeletal Class III children treated by modified maxillary protraction headgear and a chin cup: a longitudinal cephalometric appraisal. Eur. J. Orthod., Oxford, v. 15, no. 2, p. 211-221, 1993.

14. TURLEY, P. K. Orthopedic correction of Class III malocclusion with palatal expansion and custom protraction headgear. J. Clin. Orthod., Boulder, v. 22, no. 5, p. 314-325, May 1988.
Endereço para correspondência João Luiz Carlini

Rua Bruno Filgueira, 369 cj. 1402

CEP: 80.240-220 - Batel, Curitiba/PR

E-mail: joaocarlini@yahoo.com.br 